\title{
Expression status of CD44 and CD133 as a prognostic marker in esophageal squamous cell carcinoma treated with neoadjuvant chemotherapy followed by radical esophagectomy
}

\author{
KOICHI OKAMOTO, ITASU NINOMIYA, YOSHINAO OHBATAKE, ATSUSHI HIROSE, TOMOYA TSUKADA, \\ SHINICHI NAKANUMA, SEISHO SAKAI, JUN KINOSHITA, ISAMU MAKINO, KEISHI NAKAMURA, \\ HIRONORI HAYASHI, KATSUNOBU OYAMA, MASAFUMI INOKUCHI, HISATOSHI NAKAGAWARA, \\ TOMOHARU MIYASHITA, TAJIMA HIDEHIRO, HIROYUKI TAKAMURA, SACHIO FUSHIDA and TETSUO OHTA
}

Department of Gastroenterologic Surgery, Kanazawa University, Kanazawa, Ishikawa 920-8641, Japan

Received March 29, 2016; Accepted July 4, 2016

DOI: $10.3892 /$ or.2016.5133

\begin{abstract}
Cancer stem cells (CSCs) have self-renewal and pluripotency capabilities and contribute to cancer progression and chemoresistance. It has been proposed that the treatment resistance and heterogeneity of CSCs are deeply involved in the prognosis of patients with esophageal squamous cell carcinoma (ESCC). The objective of this study was to identify the influence of the expression status of the CSC markers CD44 and CD133 on chemotherapeutic efficacy and prognosis in ESCC patients who underwent radical esophagectomy after neoadjuvant chemotherapy (NAC). Endoscopically biopsied specimens taken before NAC and surgically resected specimens after NAC were immunohistochemically assessed for CD44 and CD133 expression for 47 ESCC patients who underwent NAC followed by radical esophagectomy. The correlation between CD44 and CD133 expression status and clinicopathological findings and the prognosis of ESCC patients after NAC followed by esophagectomy were analyzed. The percentages of CD44-positive cells and CD133-positive cells in specimens were increased after NAC. CD44 and CD133 expression status before NAC did not correlate with the degree of tumor progression and had no impact on the chemotherapeutic effect. However, strong expression of CD44 or CD133 and a high proportion of CD133-expressing cells before NAC were significantly associated with poorer esophageal cancer-specific survival. Patients with strong expression of CD44 or CD133 and those with a high ratio of CD133-positive tumor cells showed significantly poor prognosis regardless of the effect of chemotherapy. Multivariate analysis showed that simultaneous
\end{abstract}

Correspondence to: Dr Itasu Ninomiya, Department of Gastroenterologic Surgery, Kanazawa University, 13-1 Takaramachi, Kanazawa, Ishikawa 920-8641, Japan

E-mail: nino@staff.kanazawa-u.ac.jp

Key words: cancer stem cell, neoadjuvant chemotherapy, prognosis, esophageal squamous cell carcinoma, esophagectomy strong expression of CD44 and CD133 before NAC, a high rate of CD133-positive tumor cells before NAC, and primary tumor remission assessed by preoperative endoscopy were significant independent prognostic factors for ESCC. Our data indicate that CD44 and CD133 expression status prior to treatment dictates the malignant potential of ESCC and may be a novel predictor of recurrence and prognosis of ESCC patients after treatment.

\section{Introduction}

The Japan Clinical Oncology Group (JCOG) 9907 study demonstrated the effectiveness of resection after neoadjuvant chemotherapy (NAC) using a combination of 5-fluorouracil (5-FU) and cisplatin (CDDP) for patients with cStage II or III advanced esophageal cancer (1). On the basis of these findings, NAC followed by surgery is now a standard treatment strategy for patients with advanced esophageal squamous cell carcinoma (ESCC) in Japan's Esophagus Cancer Treatment Guidelines (2).

Although cancer stem cells (CSCs) represent only a small fraction of the cancer cluster, they have self-renewal and pluripotency capabilities similar to normal stem cells and contribute to the differentiation and proliferation of cancer cells (3). In addition, CSCs are known to be resistant to anticancer drugs and radiation. The treatment resistance and heterogeneity of CSCs are thought to play a substantial role in the prognosis of patients with ESCC.

CD44 is one of the most frequently used markers to identify a subpopulation of cells with CSC properties in various solid tumors and is broadly accepted as a marker of poor prognosis in various cancers (4-9). CD44 plays a central role in the remodeling and degradation of hyaluronan that leads to cell migration, cancer invasion, and metastasis. CD133 is another cell surface marker of CSCs and a predictor of prognosis in ESCC patients (10-12). Correlative studies of tumor specimens prior to chemotherapy can provide information on biomarkers that may predict response or resistance to chemotherapy. However, there have been few studies examining the expression pattern of both CD44 and CD133 in ESCC tissue. 
Therefore, we conducted this study to investigate the influence of a combination of the CSC markers CD44 and CD133 on the therapeutic response and prognosis of ESCC patients who underwent NAC followed by radical esophagectomy.

\section{Materials and methods}

Human tissue samples. The present study included endoscopic biopsy specimens taken before treatment and surgically resected specimens from 47 patients with ESCC who underwent video-assisted thoracoscopic esophagectomy (VATS-E) or blunt esophagectomy after preoperative chemotherapy between 2008 and 2012 at Kanazawa University Hospital. Cancer tissue specimens were collected from the patients after informed consent was obtained, in accordance with the institutional guidelines of our hospital. All patients were staged to cStage I, II, III and IV according to the 7th edition of the Tumor-Node-Metastasis (TNM) Staging system (13). The histological types of tumors were classified according to the WHO Classification of Tumors of the Digestive System, 4th edition (14). All resected primary tumors and lymph nodes were subjected to standard hematoxylin and eosin staining and classified according to the TNM classification system (13). Histologically, all of the tumors were squamous cell carcinomas (SCCs) (7 well differentiated, 18 moderately differentiated, and 22 poorly differentiated). Positive lymph node metastasis was suspected in 36 cases $(76.6 \%)$ prior to treatment and 30 cases $(63.8 \%)$ were proved to have pathological lymph node metastases. The median follow-up period was 42 months (range, 6-82 months). During this period, 20 patients $(42.6 \%)$ experienced a recurrence.

Assessment of clinical and pathological response to NAC. The clinical response of the primary tumor was evaluated by endoscopic examination according to the Response Evaluation Criteria in Solid Tumors (15). The pathological response was histopathologically diagnosed according to the evaluation criteria of the Japan Esophageal Society (16) using a 5-grade scale (grades $0,1 \mathrm{a}, 1 \mathrm{~b}, 2$ and 3 ) as follows: grade 0 , no response or almost no change in cancer cells after treatment; grade 1, slight response; grade 1a, mild response, mild change in cancer cells regardless of the area, or marked changes in cancer cells in less than one-third of total cancer cells; grade $1 b$, moderate response, marked changes in one-third or more but less than two-thirds of tumor cells; grade 2, marked response or marked changes in two-thirds or more of tumor cells; and grade 3 , no residual tumor cells, necrosis or disappearance of all tumor cells, or replacement of all cancer cells by granuloma-like and/or fibrous tissue. Patients were classified into two groups according to histopathological effect as follows: a poor response group of grades $0,1 \mathrm{a}$ and $1 \mathrm{~b}$, and a good response group of grades 2 and 3.

Immunohistochemistry. For immunohistochemical examination, $20 \%$ formalin-fixed and paraffin-embedded specimens were retrieved from the surgical pathology files of the Pathology Section of Kanazawa University Hospital, Kanazawa, Japan. The expression of CD44 and CD133 in endoscopic biopsy specimens and surgically resected ESCC specimens was examined immunohistochemically using a horseradish peroxidase (HRP)-based method. To identify the antigen in tissues, deparaffinized sections were pretreated by autoclaving in $10 \%$ citric acid buffer $\left(\mathrm{pH} \mathrm{8.0)}\right.$ at $120^{\circ} \mathrm{C}$ for $15 \mathrm{~min}$. After pretreatment with protein block serum (Dako Cytomation, Kyoto, Japan) for $10 \mathrm{~min}$ and $2 \%$ skim milk for 20 min to block nonspecific reactions, the sections were incubated with mouse anti-CD44 monoclonal antibody [diluted 1:100 in phosphate-buffered saline (PBS); R\&D Systems, Inc., Minneapolis, MN, USA] or rabbit anti-CD133 (PROM-1) polyclonal antibody (diluted 1:250 in PBS; Abnova Corporation, Taipei, Taiwan) at $4^{\circ} \mathrm{C}$ overnight. After incubation, the Envision+ polymer solution (HRP-conjugated secondary antibody; Dako Cytomation) was applied for $1 \mathrm{~h}$. The reaction products were developed in $0.02 \% 3,3$-diaminobenzidine tetrahydrochloride (DAB) solution containing $0.1 \% \mathrm{H}_{2} \mathrm{O}_{2}$. The sections were then lightly counterstained with hematoxylin and the slides were examined under a microscope (Olympus, Tokyo, Japan).

Evaluation of immunohistochemical variables. Immunohistochemical staining of CD44 and CD133 in tumor tissues was evaluated microscopically. Specimens were defined as positive for CD44 and CD133 expression when positive staining was noted in the cytoplasm and/or cell membrane of the tumor cells regardless of the strength of staining and ratio of positive cells. The interpretation of immunoreactivity was performed in a semi-quantitative manner by analyzing both the percentage of positive cells and the intensity of staining. The percentage of positive staining cells in the specimens was divided into two groups as follows: low-rate group, less than $80 \%$ of cancer cells were positive; high-rate group, more than $80 \%$ of the cancer cells were positive. The intensity of staining was divided into two groups as weak or strong. We examined the correlation between CD44 and CD133 expression status and clinicopathological factors of the tumor prior to treatment, clinicopathological therapeutic effects after NAC, and the prognosis of ESCC patients after treatment. We also evaluated changes in the proportion of CD44 and CD133-expressing tumor cells in the tissue before vs. after chemotherapy.

Statistical analysis. Results are expressed as the mean \pm standard deviation. Immunohistochemical results of CD44 and CD133 expression status before and after NAC and the correlation between the histopathological effects of chemotherapy with the clinical variables were analyzed using paired Student's t-test or Mann-Whitney U test and assessed by the chi-square test or Fisher's exact test as appropriate. The Kaplan-Meier method was used to analyze survival, and the log-rank test was used to estimate differences in survival. Prognostic factors were examined using univariate analysis, multivariate analysis, and the Cox proportional hazards regression model. Statistical significance was assumed for $\mathrm{P}<0.05$. All analyses were performed with SPSS IBM Statistics ver. 22; IBM Corp., Armonk, NY, USA).

\section{Results}

Expression of CD44 and CD133 in endoscopic biopsy specimens and resected esophageal cancer specimens. Representative expression patterns of CD44 and CD133 

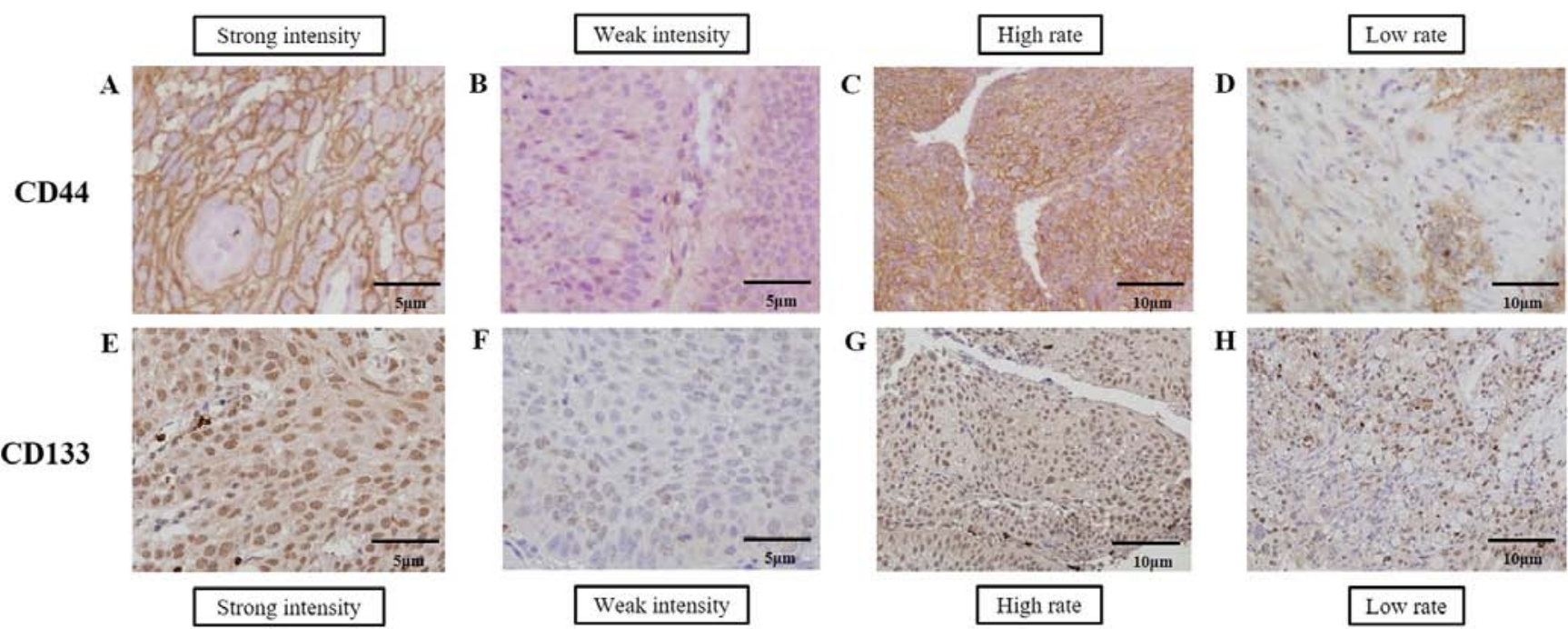

Figure 1. Representative expression patterns of CD44 and CD133 in esophageal cancer biopsy specimens. (A-D) CD44 immunoreactivity was diffusely evident in membranous and granular cytoplasmic patterns. (E-H) CD133 immunoreactivity was diffusely evident in membranous, granular cytoplasmic, and intranuclear patterns. (A) Strong intensity membranous immunostaining for CD44. (B) Weak intensity immunostaining for CD44. (C) High rate of CD44-positive cells in tumor tissue. (D) Low rate of CD44-positive cells in tumor tissue. (E) Strong intensity of nuclear and cytoplasmic immunostaining for CD133. (F) Weak intensity of immunostaining for CD133. (G) High rate of CD133-positive cells in tumor tissue. (H) Low rate of CD133-positive cells in tumor tissue.

in ESCC biopsy specimens are shown in Fig. 1. CD44 immunoreactivity was diffusely evident in membranous and granular cytoplasmic patterns (Fig. 1A and D) whereas CD133 immunoreactivity was diffusely evident in granular cytoplasmic and intranuclear patterns (Fig. 1E). Demographics of the 47 ESCC patients categorized according to CD44 and CD133 expression status in biopsied specimens before chemotherapy are shown in Table I. Strong or weak positive CD44 staining was observed in all cases and only one case showed negative staining of CD133. A single case with negative expression of CD133 was included in the weak intensity group. The level of CD44 and CD133 expression before NAC was not associated with preoperative assessment of tumor depth, lymph node metastasis, degree of tumor differentiation, and cStage (Table I).

The expression status of CD44 and CD133 in tumor cells in the biopsied specimens before NAC and the resected tissue after NAC in same patients was analyzed (Fig. 2). CD44- or CD133-positive cell were diffusely observed in the biopsied specimens before chemotherapy (Fig. 2A and C, and $E$ and $G$ ). In some cases with high chemotherapeutic effects, CD44- or CD133-positive cell clusters were observed in the necrotic and fibrotic areas of resected tissues of ESCC after NAC (Fig. 2B and F). However in the cases with poor chemotherapeutic effects, most CD44- or CD133-positive cells distributed similarly to the pre-treatment state (Fig. 2D and $\mathrm{H}$ ). There were almost no obvious changes in the intensity before and after chemotherapy. The median rate of CD44-positive and CD133-positive cells in endoscopically biopsied specimens before chemotherapy was $65.5 \pm 16.3$ and $62.3 \pm 21.6 \%$, respectively. In contrast, the median rate of CD44-positive and CD133-positive cells in resected specimens after NAC was $73.8 \pm 20.1$ and $73.8 \pm 19.6 \%$, respectively. The rates of both CD44-positive and CD133-positive cells in the tissue were significantly increased after chemotherapy $(C D 44, P=0.012$; CD133, $\mathrm{P}=0.008$ ) (Fig. 3).
Correlations between CSC marker expression before chemotherapy and clinicopathological effects of chemotherapy. There was no significant correlation between the status of each CSC marker before NAC and the clinicopathological effects of NAC (Table II). However, the histopathological effect of NAC in resected specimens was significantly correlated with clinical response after NAC, tumor depth, and cStage (Table III).

Prognostic significance of CD44 and CD133 expression. Survival analyses showed that strongly positive expression of CD44 or CD133 before NAC was significantly correlated with poor esophageal cancer-specific survival (ECSS) $(\mathrm{P}=0.003$ and $\mathrm{P}=0.024$, respectively) (Fig. 4A and $\mathrm{C}$ ). Fifteen ESCC patients with a high positive rate of CD133 expression before NAC showed significantly poorer ECSS than patients with a low positive rate of CD133 expression $(\mathrm{P}=0.003)$ (Fig. 4D). However, the positivity rate of CD44 expression before NAC had no significant prognostic impact on ECSS ( $\mathrm{P}=0.446)$ (Fig. 4B). We also analyzed the impact of combined expression of CD44 and CD133 on the outcome of ESCC and found that ESCC patients with CD44 $4^{\text {strong }} / \mathrm{CD} 133^{\text {strong }}$ expression showed significantly poorer ECSS than those with CD $44^{\text {weak }}$ or CD $44^{\text {strong }} / \mathrm{CD} 133^{\text {weak }}$ expression $(\mathrm{P}<0.001)$ (Fig. 5).

The results of univariate and multivariate survival analysis for CSC markers and each clinicopathological factor are shown in Table IV. We found that CD44 ${ }^{\text {strong }} / \mathrm{CD} 133^{\text {strong }}$ expression, higher positive rate of CD133 expression before NAC, and progressive response in the primary tumor evaluated by endoscopy were significant unfavorable prognosticators for ECSS (Table IV).

\section{Discussion}

In the present study, we found that the combination of CD44 and CD133 expression status before NAC was a novel predictor 


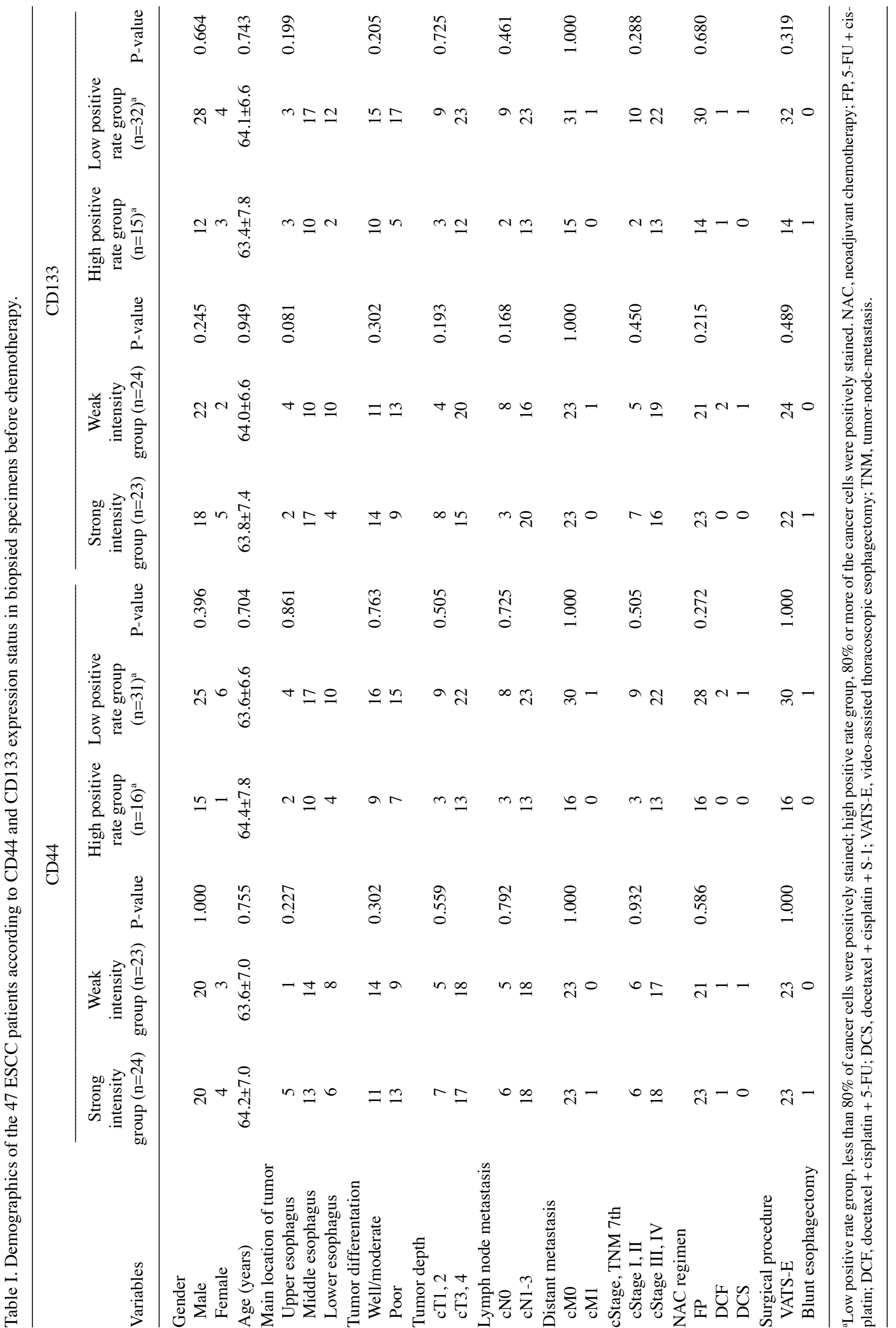



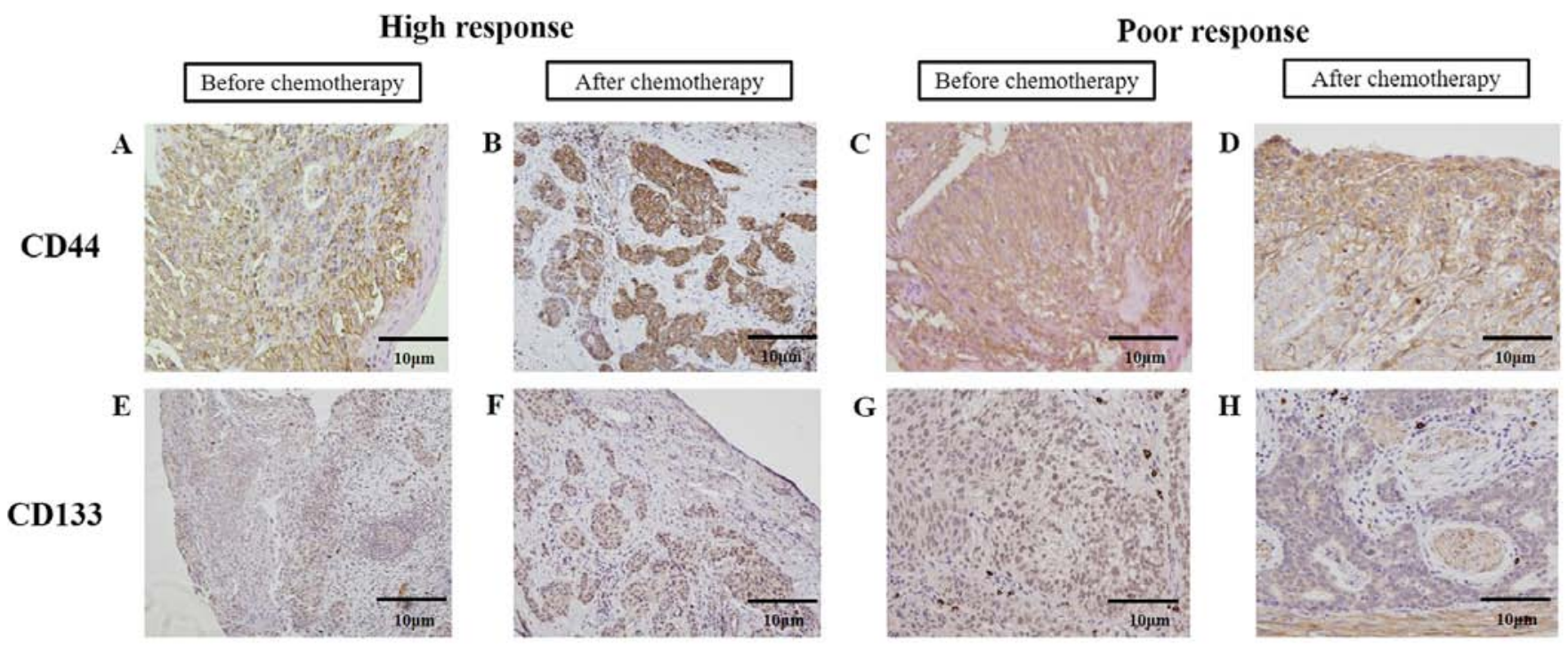

Figure 2. Changes in CD44 and CD133 expression in esophageal cancer tissues before and after chemotherapy. (A-D) Representative photomicrographs of CD44 and (E-H) CD133 immunohistochemistry (A, C, E and G) before and (B, D, F and H) after chemotherapy in the cases with high or poor therapeutic response. Immunohistochemical staining before and after chemotherapy was performed in the same patient (B, D, F and H correspond to A, C, E and G, respectively). (B and F) In the cases with high therapeutic response to chemotherapy, relatively strong intensity and high positive rate for CD44 or CD133-expressing tumor cells were observed before chemotherapy and some clusters of residual cancer cells with strong CD44 or CD133 expression were observed in tumor tissue after chemotherapy. (D and H) In the cases with poor therapeutic response to chemotherapy, strong intensity and a high positive rate for CD44 or CD133 expression were observed before chemotherapy and many CD44- or CD133-positive cells remained.
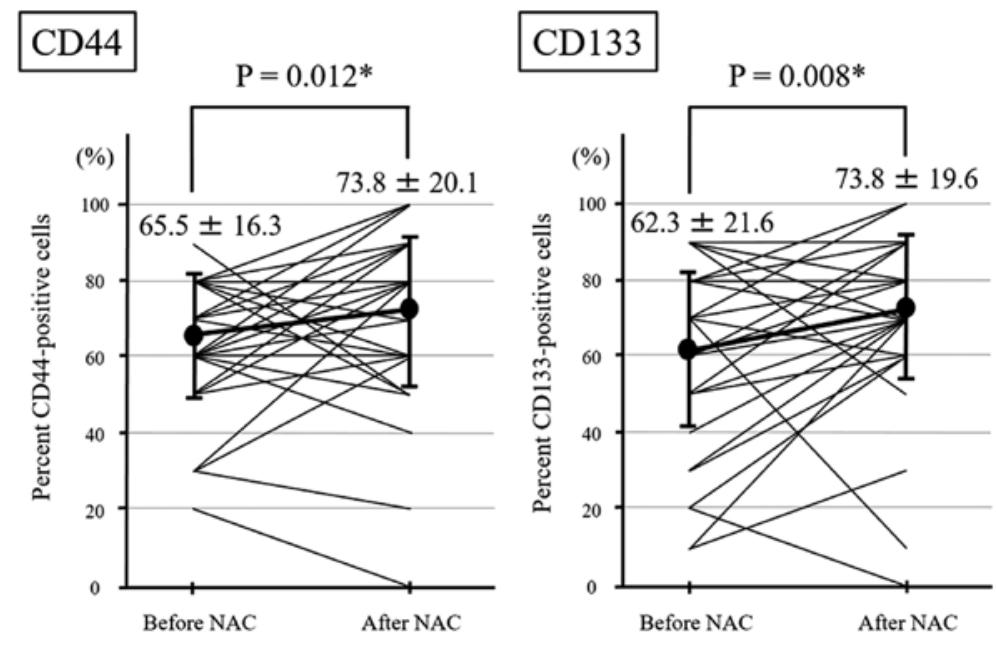

Figure 3. Changes in the proportions of CD44- and CD133-positive cells before and after chemotherapy. The proportion of cancer cells with CD44- and CD133-positive expression in resected esophageal cancer tissues after chemotherapy was significantly increased compared with that in biopsied specimens before chemotherapy.

of the prognosis of ESCC patients who underwent surgical resection after chemotherapy.

Despite the development of treatment strategies the prognosis of ESCC remains poor because of its high rate of recurrence and metastasis. The prognosis of ESCC patients cannot be accurately estimated based only on clinicopathological factors such as pretreatment TNM stage or the degree of tumor differentiation; it is also critical to classify the effectiveness of chemotherapy and the probability of recurrence or metastasis in pretreatment ESCC patients.

It has been reported that NAC has numerous advantages in various solid tumors and pathological response data for NAC are thought to be very beneficial as surrogate markers for long-term clinical outcome $(17,18)$. To estimate the therapeutic response and prognosis after therapy, we investigated the CSC profile in primary ESCC tissue because CSC-like cells are known to be resistant to chemotherapy or radiation and are often characterized by elevated expression of the stem cell surface markers CD44 and CD133 (18-21). Stem cells are characterized by the properties of self-renewal and pluripotency. CSC-like cells are thought to be tumor-initiating cells (TICs) and exhibit characteristics of low rates of proliferation, a high capacity for self-renewal, and a propensity to differentiate into actively proliferating cancer cells $(3,22)$. To date, several markers have been used to identify CSCs, including CD44, CD133, aldehyde dehydrogenase 1 (ALDH1), 


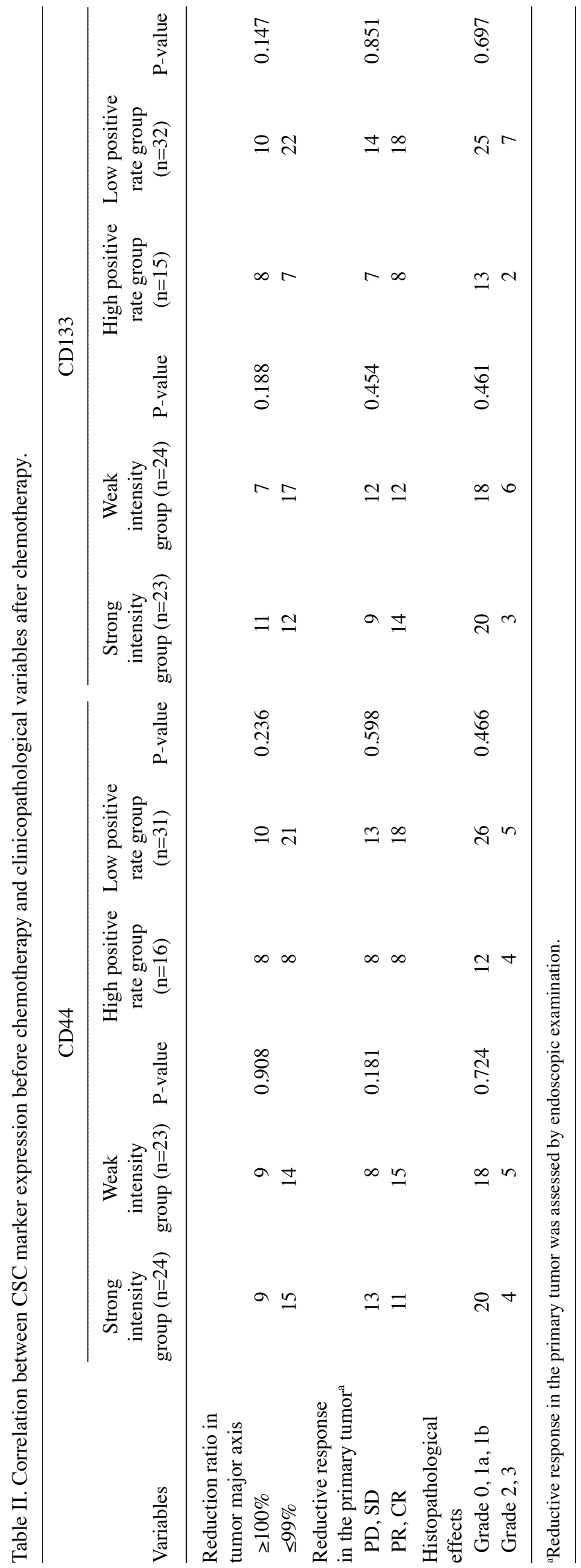


Table III. Correlation between the histopathological effects of chemotherapy and the clinical variables.

\begin{tabular}{|c|c|c|c|}
\hline & \multicolumn{3}{|c|}{ Histopathological effects } \\
\hline Variables & $\begin{array}{c}\text { Grades } 0,1 \mathrm{a} \\
\text { and } 1 \mathrm{~b}(\mathrm{n}=38)\end{array}$ & $\begin{array}{c}\text { Grades } 2 \\
\text { and } 3(n=9)\end{array}$ & P-value \\
\hline
\end{tabular}

\begin{tabular}{|c|c|c|c|}
\hline \multicolumn{4}{|l|}{$\begin{array}{l}\text { Reduction ratio in } \\
\text { tumor major axis }\end{array}$} \\
\hline$\leq 99 \%$ & 21 & 8 & 0.124 \\
\hline$\geq 100 \%$ & 17 & 1 & \\
\hline \multicolumn{4}{|c|}{$\begin{array}{l}\text { Reductive response in } \\
\text { the primary tumor }\end{array}$} \\
\hline $\mathrm{PR}, \mathrm{CR}$ & 18 & 8 & $0.030^{\mathrm{a}}$ \\
\hline $\mathrm{PD}, \mathrm{SD}$ & 20 & 1 & \\
\hline \multicolumn{4}{|l|}{ Clinical parameters } \\
\hline \multicolumn{4}{|l|}{$\mathrm{cT}$} \\
\hline $\mathrm{cT} 1,2$ & 7 & 5 & $0.035^{\mathrm{a}}$ \\
\hline cT3, 4 & 31 & 4 & \\
\hline \multicolumn{4}{|l|}{$\mathrm{cN}$} \\
\hline cNO & 8 & 3 & 0.419 \\
\hline $\mathrm{cN} 1-3$ & 30 & 6 & \\
\hline \multicolumn{4}{|l|}{ cStage } \\
\hline cStage I, II & 6 & 6 & $0.005^{\mathrm{a}}$ \\
\hline cStage III, IV & 32 & 3 & \\
\hline \multicolumn{4}{|c|}{ Tumor differentiation } \\
\hline Well/moderately & 21 & 4 & 0.715 \\
\hline Poor & 17 & 5 & \\
\hline
\end{tabular}

The histopathological effect of NAC in resected specimens was significantly correlated with clinical reductive response after NAC, tumor depth, and cStage. NAC, neoadjuvant chemotherapy; ${ }^{\mathrm{a}} \mathrm{P}<0.05$. ${ }^{\mathrm{b}}$ Reductive response in the primary tumor was assessed by endoscopical examination.

adenosine triphosphate-binding cassette superfamily $G$ member 2 (ABCG2), and Bmi-1 (4,11,23-27). Among these, CD44 and CD133 are known to be representative markers of CSCs. In the present study, we performed immunohistochemical analyses for the detection and the assessment of CSC-like cells by examining CD44 and CD133 expression in ESCC tissues before and after chemotherapy.

CD44 is the transmembrane adhesion receptor for hyaluronan and plays a central role in the remodeling and degradation processes of hyaluronan that lead to cell migration, as well as to cancer invasion and metastasis through cell-cell and cell-extracellular matrix adhesion. Many researchers have demonstrated that CSCs express CD44-related surface markers. CD44 is one of the most frequently used markers to identify a subpopulation of cells with CSC properties in solid tumors, including colon cancer, head and neck SCC, lung cancer, gastric cancer, pancreatic cancer, and ESCC, and is broadly accepted as a marker of poor prognosis in various cancers (4-9). Biddle et al (28) reported that CD44 and epithelial cell adhesion molecule (EpCAM) expression in CSCs correlated with the switch between mesenchymal phenotype and epithelial phenotype in association with the epithelial-to-mesenchymal transition (EMT) in head and neck SCC. It has also been reported that CD44-positive CSCs exhibit molecular characteristics associated with EMT in SCC (29-31). CD44 downregulates E-cadherin expression, upregulates vimentin and matrix metalloproteinase (MMP), and inhibits the formation of membrane-associated E-cadherin- $\beta$-catenin complex, resulting in cell invasion and migration (30). It has also been reported that CD44 is a novel surface marker of TICs, a subpopulation of cells with the ability to self-renew as well as drive the initiation and progression of cancer $(9,22)$. These findings suggested that CD44 expression may correlate with cancer cell viability, postoperative recurrence, and metastasis of solid tumors through the process of EMT and resistance to chemotherapy and radiation.

CD133 protein encoded by the PROM1 gene is known as a cell surface marker of stem cells, including embryonic stem cells and progenitor cells $(32,33)$. It has been reported that CD133 is also expressed on CSCs, such as leukemic stem cells and liver CSCs $(10,11)$. It has also been reported that CD133 immunoreactivity is a good predictor of prognosis in ESCC patients and that CD133 may play a role in the regulation of the tumor cell cycle through p27 and p16 in ESCC (12). In the present study we observed strongly positive expression of CD44 and CD133 in 51.1 and 48.9\%, respectively, of the biopsied specimens before NAC. Little change in the intensity of CD44 and CD133 expression was observed in resected specimens after NAC, but enrichment for CD44- or CD133-positive cells was observed in post-NAC tumor specimens compared with pre-NAC specimens. The results of survival analyses showed that strongly positive expression of CD44 or CD133 and higher positive rate of CD133 before NAC were significantly correlated with a poorer prognosis. In addition, combination analysis of CD44 and CD133 was useful for predicting relapse-free survival. Multivariate analysis showed that simultaneous strong expression of CD44 and CD133, a high positive rate of CD133-expressing tumor cells, and a progressive response in the primary tumor were independent prognosticators for ECSS.

In the present study, CD44 and CD133 positive ratio before chemotherapy was 65.5 and $62.3 \%$, respectively. We tried to examine the proportion and distribution of CSCs in tumor tissue by CD44 and CD133 immunoreactivity. It is considered that CD44- and/or CD133-positive cells does not represent actual CSCs, because the ratio of CD44- and/or CD133-positive cells is much higher than the actual proportion of exact CSCs in tumor tissue. However, it is considered that CD44 and/or CD133-expressing tumor cells have the characteristics of cancer stem-like cell properties. Histological chemo-resistance and prognostic significance of these markers before treatment may support this hypothesis. Generally, previous studies have reported that the histopathological response reflects the prognosis of solid cancers and that CSC marker expression was correlated with treatment resistance (17). Aomatsu et al (18) reported that the $\mathrm{cCR}$ rate of $\mathrm{CD} 133$-positive tumors was significantly lower than that of CD133-negative tumors and that CD133 expression before NAC was an independent predictive factor for pCR in breast cancer. Other studies also showed that higher expression of CD133 was a poor prognostic factor and 
Table IV. Univariate and multivariate analyses of clinicopathological variables associated with esophageal cancer-specific survival.

\begin{tabular}{|c|c|c|c|c|c|c|}
\hline \multirow[b]{2}{*}{ Variables } & \multicolumn{3}{|c|}{ Univariate } & \multicolumn{3}{|c|}{ Multivariate } \\
\hline & HR & $95 \% \mathrm{CI}$ & P-value & HR & $95 \% \mathrm{CI}$ & P-value \\
\hline \multicolumn{7}{|l|}{ CSC markers } \\
\hline $\begin{array}{l}\text { Intensity of CD expression } \\
\text { before NAC } \\
\left(\mathrm{CD} 44^{\text {strong}} / \mathrm{CD} 133^{\text {strong }} \text { vs. other }\right)\end{array}$ & 5.618 & $1.980-15.87$ & $0.001^{\mathrm{a}}$ & 25.641 & $3.021-200$ & $0.003^{\mathrm{a}}$ \\
\hline $\begin{array}{l}\text { Positive rate of CD expression } \\
\text { before NAC } \\
\left(\mathrm{CD} 133^{\text {high }} \text { vs. CD133 }{ }^{\text {low }}\right)\end{array}$ & 5.015 & $1.060-23.716$ & $0.042^{\mathrm{a}}$ & 6.778 & $1.327-34.624$ & $0.021^{\mathrm{a}}$ \\
\hline \multicolumn{7}{|l|}{ Response to NAC } \\
\hline $\begin{array}{l}\text { Reduction ratio in major axis } \\
\text { of the tumor ( } \geq 100 \mathrm{vs.} \leq 99 \% \text { ) }\end{array}$ & 4.202 & $1.067-16.393$ & $0.040^{\mathrm{a}}$ & & & \\
\hline $\begin{array}{l}\text { Reductive response in the primary } \\
\text { tumor }(\mathrm{PD}, \mathrm{SD} \text { vs. } \mathrm{PR}, \mathrm{CR})^{\mathrm{b}}\end{array}$ & 6.329 & $1.337-29.412$ & $0.020^{\mathrm{a}}$ & 10.101 & $1.799-55.556$ & $0.009^{\mathrm{a}}$ \\
\hline $\begin{array}{l}\text { Clinicopathological parameters } \\
\mathrm{cT}(3,4 \text { vs. } 1,2) \\
\mathrm{cN}(\geq 1 \text { vs. } 0)\end{array}$ & $\begin{array}{l}1.181 \\
5.618\end{array}$ & $\begin{array}{l}0.591-2.363 \\
0.226-142.86\end{array}$ & $\begin{array}{l}0.638 \\
0.292\end{array}$ & & & \\
\hline $\begin{array}{l}\text { Degree of differentiation } \\
\text { Poor vs. well/moderate }\end{array}$ & 1.200 & $0.644-2.237$ & 0.566 & & & \\
\hline $\begin{array}{l}\text { Histopathological effects } \\
\text { Grade }(0,1 \mathrm{a}, 1 \mathrm{~b} \text { vs. } 2,3)\end{array}$ & 1.101 & $0.502-2.421$ & 0.809 & & & \\
\hline cStage (III, IV vs. I, II) & 1.058 & $0.482-2.326$ & 0.888 & & & \\
\hline
\end{tabular}

HR, hazard ratio; 95\% CI, 95\% confidence interval; NAC, neoadjuvant chemotherapy; ${ }^{a}<<0.05$. ${ }^{b}$ Reductive response in the primary tumor was assessed by endoscopic examination.
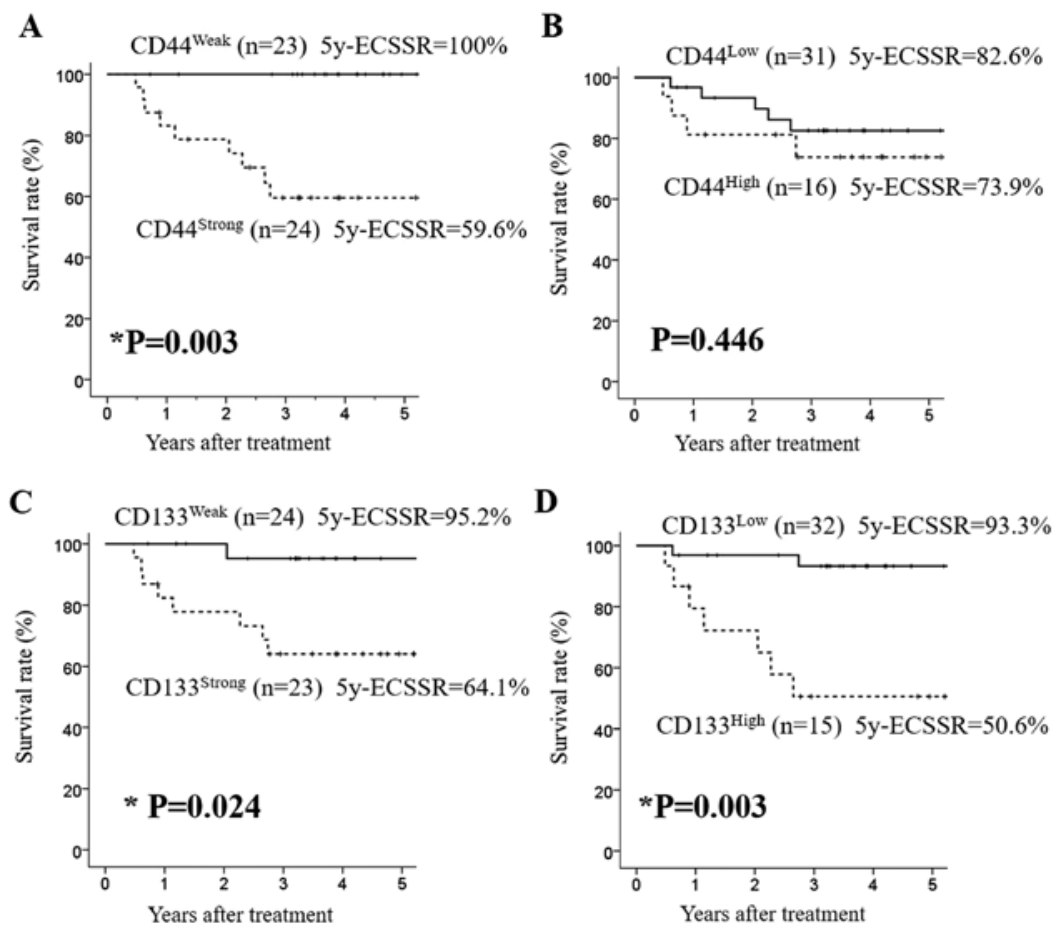

Figure 4. Esophageal cancer-specific survival curves of esophageal cancer patients categorized according to CD44 and CD133 expression status in biopsied specimen before chemotherapy. (A and B) CD44 and (C and D) CD133 expression status was classified according to the (A and C) intensity and (B and D) positive ratio. A case with negative expression of CD133 was included in the weak intensity group. The population with strong intensity of CD44 or CD133 expression and a high positive rate of CD133 expression was significantly correlated with worse prognosis. 5y-ECSSR, 5-year esophageal cancer-specific survival rate. ${ }^{*} \mathrm{P}<0.05$. 


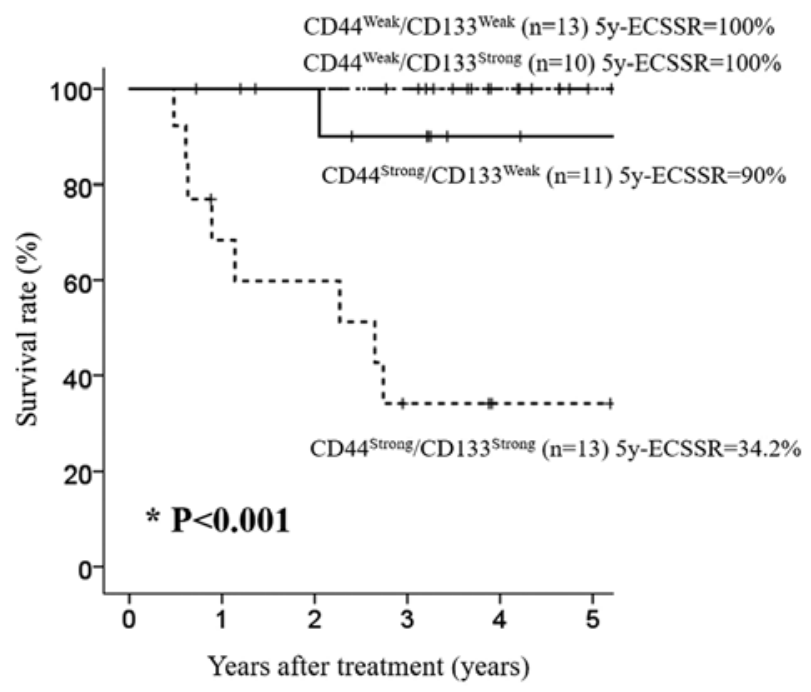

Figure 5. Esophageal cancer-specific survival curves of esophageal cancer patients categorized according to combined CD44 and CD133 expression status in biopsied specimen tissues before chemotherapy. The cases with CD $44^{\text {strong }} / \mathrm{CD} 133^{\text {strong }}$ expression showed significantly poorer prognosis than those with other combinations of CD44 and CD133 status ( $\mathrm{P}<0.001)$ A single case with negative expression of CD133 was included in the weak intensity group.

was correlated with resistance to chemotherapy and radiation in colorectal cancer $(23,34)$. Sahlberg et al (21) demonstrated that colon cancer cells with high CD133 and CD44 expression were associated with AKT expression and increased radiation resistance, and that the various AKT isoforms had different effects on the expression of CSC markers. In the present study, we found that ESCC patients with strong expression of CSC markers in pre-NAC specimens had a poor prognosis regardless of the effect of chemotherapy. From these results, it was inferred that non-CSC like cells were consigned to cell death after chemotherapy whereas highly chemoresistant CSC marker-positive cells survived. Our finding that patients with CD44 ${ }^{\text {strong }}$ or CD133 $3^{\text {strong }}$ expression had poorer prognosis (Fig. 4) was consistent with the results of previous studies $(18,23,34)$. In addition, our present study also revealed the value of the combined CD44 and CD133 expression status as a more sensitive prognostic predictor in ESCC patients than CD44 or CD133 expression status alone.

Assessment of the chemoresistance of each tumor is very important for the selection of effective treatment methods, but no definitive biomarkers have been shown to predict the therapeutic effects. Correlative studies of tumor specimens before and after NAC may provide further information on markers that predict the response or resistance to chemotherapy. However, only a few studies have previously examined the histopathological responses in tumors before and after chemotherapy (35). The present study investigating the expression status of CSCs markers before and after chemotherapy provides the prognostic significance of CSC marker expression status before chemotherapy, indicating that the high population of CSC-like tumor cells shows chemoresistance.

Our study revealed that CSCs that were originally present in tumor tissues reflect the high risk of metastasis and recurrence and the poor prognosis of ESCC patients because of the refractoriness of these cells to chemotherapy. Deregulation of the refractoriness of CSCs to anticancer drugs may have an immense clinical benefit on the prognosis of patients with ESCC.

In the future, it will be possible to predict the optimal combination of anticancer drugs using the expression of a combination of key proteins in tumor tissues as a criterion to decide treatment selection and predict adverse effects. ALDH1 has been indicated as a possible candidate for a CSC marker (23). The expression of ALDH1 was correlated with clinicopathological factors and clinical outcomes of patients with ESCC (24). ALDH1 was also found to be a more significantly predictive marker than $\mathrm{CD} 44^{+} / \mathrm{CD} 24^{-}$for the identification of breast CSCs in terms of resistance to chemotherapy (35). In a study of the prognostic value of ABCG2 and CD133 expression in ESCC patients, ABCG2 expression was found to be significantly correlated with survival whereas CD133 expression was not (26). If it is possible to use combinations of various biomarkers for more effective prediction of therapeutic effects, we may expect the development of tailor-made treatments and an improvement in prognosis in not only patients with ESCC, but also in those with various other tumors. Understanding the molecular biology of CSC-associated molecules is now essential for the development of more effective cancer treatments. Moreover, an understanding of CSC characteristics associated with the development of cancer and carcinogenesis may have clinical applications for the prediction of refractoriness to radiation or chemotherapy. Finally, although CSCs are considered to play a pivotal role in tumor progression and the metastatic process, other mechanisms that impair the malignant potential and chemoresistance should also be investigated.

In conclusion, combination analysis of CD44 and CD133 expression can be useful to predict prognosis of ESCC patients. Immunohistochemical assessment of CSC markers in pretreatment ESCC specimens may help physicians determine the malignant potential of an individual patient's tumor and their likely prognosis.

\section{References}

1. Ando N, Kato H, Igaki H, Shinoda M, Ozawa S, Shimizu H, Nakamura T, Yabusaki H, Aoyama N, Kurita A, et al: A randomized trial comparing postoperative adjuvant chemotherapy with cisplatin and 5-fluorouracil versus preoperative chemotherapy for localized advanced squamous cell carcinoma of the thoracic esophagus (JCOG9907). Ann Surg Oncol 19: 68-74, 2012.

2. Kuwano H, Nishimura Y, Oyama T, Kato H, Kitagawa Y, Kusano M, Shimada H, Takiuchi H, Toh Y, Doki Y, et al: Guidelines for Diagnosis and Treatment of Carcinoma of the Esophagus April 2012 edited by the Japan Esophageal Society. Esophagus 12: 1-30, 2015.

3. Reya T, Morrison SJ, Clarke MF and Weissman IL: Stem cells, cancer, and cancer stem cells. Nature 414: 105-111, 2001.

4. Wang C, Xie J, Guo J, Manning HC, Gore JC and Guo N: Evaluation of CD44 and CD133 as cancer stem cell markers for colorectal cancer. Oncol Rep 28: 1301-1308, 2012.

5. Chen J, Zhou J, Lu J, Xiong H, Shi X and Gong L: Significance of CD44 expression in head and neck cancer: A systemic review and meta-analysis. BMC Cancer 14: 15, 2014.

6. Zhao S, He JL, Qiu ZX, Chen NY, Luo Z, Chen BJ and Li WM: Prognostic value of CD44 variant exon 6 expression in non-small cell lung cancer: A meta-analysis. Asian Pac J Cancer Prev 15: 6761-6766, 2014.

7. Nosrati A, Naghshvar F and Khanari S: Cancer stem cell markers CD44, CD133 in primary gastric adenocarcinoma. Int J Mol Cell Med 3: 279-286, 2014. 
8. Mizukami T, Kamachi H, Mitsuhashi T, Tsuruga Y, Hatanaka Y, Kamiyama T, Matsuno Y and Taketomi A: Immunohistochemical analysis of cancer stem cell markers in pancreatic adenocarcinoma patients after neoadjuvant chemoradiotherapy. BMC Cancer 14: 687, 2014.

9. Zhao JS, Li WJ, Ge D, Zhang PJ, Li JJ, Lu CL, Ji XD, Guan DX, Gao H, Xu LY, et al: Tumor initiating cells in esophageal squamous cell carcinomas express high levels of CD44. PLoS One 6: e21419, 2011

10. Horn PA, Tesch H, Staib P, Kube D, Diehl V and Voliotis D: Expression of AC133, a novel hematopoietic precursor antigen, on acute myeloid leukemia cells. Blood 93: 1435-1437, 1999.

11. Suetsugu A, Nagaki M, Aoki H, Motohashi T, Kunisada T and Moriwaki H: Characterization of $\mathrm{CD}_{133^{+}}$hepatocellular carcinoma cells as cancer stem/progenitor cells. Biochem Biophys Res Commun 351: 820-824, 2006.

12. Okamoto H, Fujishima F, Nakamura Y, Zuguchi M, Ozawa Y, Takahashi Y, Miyata G, Kamei T, Nakano T, Taniyama Y, et al: Significance of CD133 expression in esophageal squamous cell carcinoma. World J Surg Oncol 11: 51, 2013.

13. Sobin L, Gospodarowicz M and Wittekind C (eds): TNM Classification of Malignant Tumors. 7th edition. Wiley-Blackwell, Oxford, 2010.

14. Fléjou JF: WHO Classification of digestive tumors: the fourth edition. Ann Pathol 31 (Suppl 5): S27-S31, 2011 (In French).

15. Therasse P, Arbuck SG, Eisenhauer EA, Wanders J, Kaplan RS, Rubinstein L, Verweij J, Van Glabbeke M, van Oosterom AT, Christian MC, et al: New guidelines to evaluate the response to treatment in solid tumors. European Organization for Research and Treatment of Cancer, National Cancer Institute of the United States, National Cancer Institute of Canada. J Natl Cancer Inst 92: 205-216, 2000.

16. Japan Esophageal Society: Japanese Classification of Esophageal Cancer, tenth edition: part I. Esophagus 6: 1-25, 2009.

17. Meredith KL, Weber JM, Turaga KK, Siegel EM, McLoughlin J, Hoffe S, Marcovalerio M, Shah N, Kelley S and Karl R: Pathologic response after neoadjuvant therapy is the major determinant of survival in patients with esophageal cancer. Ann Surg Oncol 17: 1159-1167, 2010.

18. Aomatsu N, Yashiro M, Kashiwagi S, Takashima T, Ishikawa T, Ohsawa M, Wakasa K and Hirakawa K: CD133 is a useful surrogate marker for predicting chemosensitivity to neoadjuvant chemotherapy in breast cancer. PLoS One 7: e45865, 2012.

19. Lee HH, Seo KJ, An CH, Kim JS and Jeon HM: CD133 expression is correlated with chemoresistance and early recurrence of gastric cancer. J Surg Oncol 106: 999-1004, 2012.

20. Piao LS, Hur W, Kim TK, Hong SW, Kim SW, Choi JE, Sung PS, Song MJ, Lee BC, Hwang D, et al: CD133+ liver cancer stem cells modulate radioresistance in human hepatocellular carcinoma. Cancer Lett 315: 129-137, 2012.

21. Sahlberg SH, Spiegelberg D, Glimelius B, Stenerlöw B and Nestor M: Evaluation of cancer stem cell markers CD133, CD44, CD24: Association with AKT isoforms and radiation resistance in colon cancer cells. PLoS One 9: e94621, 2014

22. Pardal R, Clarke MF and Morrison SJ: Applying the principles of stem-cell biology to cancer. Nat Rev Cancer 3: 895-902, 2003
23. Zhou F, Mu YD, Liang J, Liu ZX, Chen HS and Zhang JF: Expression and prognostic value of tumor stem cell markers ALDH1 and CD133 in colorectal carcinoma. Oncol Lett 7 : 507-512, 2014

24. Wang Y, Zhe H, Gao P, Zhang N, Li G and Qin J: Cancer stem cell marker ALDH1 expression is associated with lymph node metastasis and poor survival in esophageal squamous cell carcinoma: A study from high incidence area of northern China. Dis Esophagus 25: 560-565, 2012.

25. Ginestier C, Hur MH, Charafe-Jauffret E, Monville F, Dutcher J, Brown M, Jacquemier J, Viens P, Kleer CG, Liu S, et al: ALDH1 is a marker of normal and malignant human mammary stem cells and a predictor of poor clinical outcome. Cell Stem Cell 1: 555-567, 2007.

26. Hang D, Dong HC, Ning T, Dong B, Hou DL and Xu WG: Prognostic value of the stem cell markers CD133 and ABCG2 expression in esophageal squamous cell carcinoma. Dis Esophagus 25: 638-644, 2012.

27. Shien K, Toyooka S, Ichimura K, Soh J, Furukawa M, Maki Y, Muraoka T, Tanaka N, Ueno T, Asano H, et al: Prognostic impact of cancer stem cell-related markers in non-small cell lung cancer patients treated with induction chemoradiotherapy. Lung Cancer 77: 162-167, 2012.

28. Biddle A, Liang X, Gammon L, Fazil B, Harper LJ, Emich H, Costea DE and Mackenzie IC: Cancer stem cells in squamous cell carcinoma switch between two distinct phenotypes that are preferentially migratory or proliferative. Cancer Res 71: 5317-5326, 2011

29. Geng S, Guo Y, Wang Q, Li L and Wang J: Cancer stem-like cells enriched with CD29 and CD44 markers exhibit molecular characteristics with epithelial-mesenchymal transition in squamous cell carcinoma. Arch Dermatol Res 305: 35-47, 2013.

30. Le Bras GF, Allison GL, Richards NF, Ansari SS, Washington MK and Andl CD: CD44 upregulation in E-cadherin-negative esophageal cancers results in cell invasion. PLoS One 6 : e27063, 2011.

31. Chen C, Zimmermann M, Tinhofer I, Kaufmann AM and Albers AE: Epithelial-to-mesenchymal transition and cancer stem(-like) cells in head and neck squamous cell carcinoma. Cancer Lett 338: 47-56, 2013

32. Yin AH, Miraglia S, Zanjani ED, Almeida-Porada G, Ogawa M, Leary AG, Olweus J, Kearney J and Buck DW: AC133, a novel marker for human hematopoietic stem and progenitor cells. Blood 90: 5002-5012, 1997.

33. Katoh $\mathrm{Y}$ and Katoh M: Comparative genomics on PROM1 gene encoding stem cell marker CD133. Int J Mol Med 19: 967-970, 2007.

34. Jao SW, Chen SF, Lin YS, Chang YC, Lee TY, Wu CC, Jin JS and Nieh S: Cytoplasmic CD133 expression is a reliable prognostic indicator of tumor regression after neoadjuvant concurrent chemoradiotherapy in patients with rectal cancer. Ann Surg Oncol 19: 3432-3440, 2012.

35. Tanei T, Morimoto K, Shimazu K, Kim SJ, Tanji Y, Taguchi T, Tamaki Y and Noguchi S: Association of breast cancer stem cells identified by aldehyde dehydrogenase 1 expression with resistance to sequential Paclitaxel and epirubicin-based chemotherapy for breast cancers. Clin Cancer Res 15: 4234-4241, 2009. 